\title{
Alterations of Erythrocyte Superoxide Dismutase activity in patients suffering from asthma attacks
}

\author{
K. Katsoulis¹, T. Kontakiotis², S. Gerou1, M. Kougioulis ${ }^{1}$, \\ H. Lithoxopoulou2, D. Papakosta²
}

ABSTRACT: Alterations of Erythrocyte Superoxide Dismutase activity in patients suffering from asthma attacks. K. Katsoulis, T. Kontakiotis, S. Gerou, M. Kougioulis, H. Lithoxopoulou, D. Papakosta.

Background. Oxidant-antioxidant imbalance may play an important role in the development and progression of bronchial asthma. However, the role of blood antioxidants especially in asthma exacerbation has not been fully discussed.

Objective. This study examines a part of the intracellular antioxidant defense mechanism in asthmatic patients admitted to hospital due to severe exacerbation of their disease.

Methods. Peripheral blood Erythrocyte Superoxide Dismutase (SOD) activity was measured in 38 patients (33 men - 5 women, with a mean age of $56 \pm 2.8 \mathrm{yrs}$ ), using a colorimetric method. On the days of admission and discharge the Forced Expiratory Volume in 1 second $\left(\mathrm{FEV}_{1}\right)$ and the Partial arterial Oxygen pressure $\left(\mathrm{PaO}_{2}\right)$ were recorded and correlated with SOD activity at the same time.
Results. A statistically significant decrease of SOD activity was observed on the day of admission compared to SOD activity on the day of discharge $(43.64 \pm 31.78 \mathrm{vs}$. 96.16 \pm 54.05 units $/ \mathrm{ml}, p<0.001$ ), suggesting the presence of oxidative stress during an asthma attack. A statistically significant correlation was observed between $\mathrm{FEV}_{1}$ on admission and SOD activity at the same time $(r=0.57, p<0.001)$. Furthermore, SOD activity on admission was correlated with $\mathrm{PaO}_{2}$ on discharge $(r=0.55, p<0.001)$, as well as SOD on discharge with $\mathrm{PaO}_{2}$ on discharge $(r=0.53, p=0.001)$.

Conclusions. Decreased systemic erythrocyte SOD activity was observed during asthma attacks. This activity was correlated with severity criteria such as FEV $_{1}$ and $\mathrm{PaO}_{2}$. Therefore, it seems that measurement of SOD activity could be a useful tool in the evaluation of an asthma attack. The supplementary administration of antioxidants in the future needs further clarification. Monaldi Arch Chest Dis 2010; 73: 3, 99-104.

Keywords: Oxidative stress, Antioxidants, Superoxide Dismutase, Asthma exacerbation, FEV . $_{\text {. }}$

1 Pulmonary Department \& Microbiology Laboratory, General Army Hospital, Thessaloniki,

2 Pulmonary Department, Aristotle University, Thessaloniki, Greece.

Correspondence: Theodore Kontakiotis, Perikleous Kanda 13, Panorama, Thessaloniki, GR 55236, Greece; e-mail: kontak@auth.gr

\section{Introduction}

In recent years, oxidative stress has been increasingly recognised as one of the major factors contributing to the chronic inflammatory process of asthma [1-3]. Furthermore, there has been considerable interest in the hypothesis that an oxidant/antioxidant imbalance may be important in the pathogenesis of asthma [4-6]. Oxygen radicals can be generated not only from incomplete reduction of oxygen to water during normal aerobic metabolism, but also from oxidative burst of activated inflammatory cells [7] and exogenous oxidants, such as environmental pollutants, cigarette smoke, drugs, chemotherapeutic agents and hyperoxia. The result is the production of molecules, which are either released into the cell or penetrate the cell membrane and are transferred to other parts of the body.

Oxygen free radicals are scavenged by antioxidants and antioxidant enzymes. One of the first defense lines includes enzymes, such as the Superoxide Dismutase (SOD), which takes part in the conversion of superoxide anion into hydrogen perox- ide and molecular oxygen. There are three forms of SOD, according to the metal contained in its molecule (copper/zinc, iron, manganese) [8]. Erythrocytes are rich in antioxidants such as SOD, catalase and components of the glutathione redox cycle. The ability of an individual to counteract the damaging effects of free radical assault depends on the antioxidant capacity in an organ or body fluid [9].

Several reports have found alterations in different antioxidants in asthma. In a stable condition, erythrocyte and serum SOD activity is higher in asthmatic patients than in normal subjects $[10,11]$. Most of the studies so far have focused on the oxidant/antioxidant imbalance that occurs in stable asthma. The effects of exacerbations have received far less attention. In a previous study of our team it was observed that extracellular antioxidant capacity, as assessed by serum total antioxidant status, was decreased in patients with severe exacerbation of asthma and correlated with the severity of the disease [12].

The aim of the present study was to investigate a main part of the intracellular antioxidant defense, through erythrocyte SOD activity, in asthmatic pa- 
tients with severe exacerbation of their disease, by measuring the red blood cells $\mathrm{Cu} / \mathrm{Zn}$-SOD activity. Additionally, we investigated the probable correlation between SOD activity and clinical or laboratory findings which are used for the evaluation of the severity of asthma.

\section{Materials and methods}

\section{Patients}

Thirty eight patients (33 men - 5 women) with a mean age of $56 \pm 2.8$ years were studied. All patients were admitted to Pulmonary Departments of two General Hospitals due to severe exacerbation of bronchial asthma. The diagnosis of asthma was based on symptoms and reversibility of airflow limitation by bronchodilators according to GINA guidelines [13]. Their medication prior to admission consisted of $\beta_{2}$ agonists or inhaled steroids or a combination of both. None of the patients was receiving oral steroids for at least one month prior to the study entry or any kind of medication with known antioxidant properties, such as trimetazidine, non steroidal anti-inflammatory drugs or acetylcysteine. None of the patients had blood glucose levels $>150 \mathrm{mg} / \mathrm{dl}$ that could increase the production of free radicals due to auto-oxidation of glucose. All patients were non-smokers. Seventeen of them had a history of atopy, defined as giving a positive wheal and flare response to skin prick testing with several common allergens.

The entry criteria were based upon low Forced Expiratory Volume in 1 second $\left(\mathrm{FEV}_{1}\right)(<60 \%$ predicted) or severe hypoxemia [Partial arterial pressure of Oxygen $\left.-\mathrm{PaO}_{2}<60 \mathrm{mmHg}(8 \mathrm{kPa})\right]$ or presence of at least one severity criterion, such as need to be seated, difficulty to speak, use of accessory respiratory muscles, respiratory rate $>30$ breaths $/ \mathrm{min}$, pulse rate $>120 / \mathrm{min}$, pulsus paradoxus, and also lack of clinical improvement despite intensive medical treatment at the Emergency Department. The most frequent cause of exacerbation of the disease was upper or lower respiratory tract infection.

\section{Study Design}

On the day of admission venous blood was drawn for complete blood count and serum chemistries before any drug administration. Also, partial arterial pressure of oxygen in room air was measured $\left(\mathrm{PaO}_{2}\right)$. A venous $5 \mathrm{ml}$ sample was sent to the Microbiology Laboratory in a heparinised syringe for immediate SOD activity measurement.

All patients were treated with nebulised beta $2^{-}$ agonists as well as parenteral steroids at the same dose (40 mg Methylprednisolone). The discharge criteria were based upon the combination of improved $\mathrm{POa}_{2}[>70 \mathrm{mmHg}(9.3 \mathrm{kPa})]$, normal respiratory rate and pulse rate, clinical improvement and improvement of $\mathrm{FEV}_{1}$ ( $>60 \%$ predicted).

On the day of discharge a venous sample was also drawn for a second measurement of SOD activity.

The study had the approval of the institutional committee on human experimentation.

\section{Laboratory Methods}

A Ransod kit purchased from Randox Ltd, Crumlin, Co Antrim, UK was used to measure SOD activity based on a colorimetric method. This method employs xanthine and xanthine oxidase to generate superoxide radicals, which react with 2-(4iodophenyl)-3-(4-nitrophenol)-5-phenyltetrazolium chloride (I.N.T.) to form a red formazan dye. The superoxide dismutase activity is then measured by the degree of inhibition of this reaction.

A $5 \mathrm{ml}$ sample of heparinised blood was centrifuged for $10 \mathrm{~min}$ in 3,000 r.p.m., plasma was removed, red cells were washed with $0,9 \%$ sodium chloride, cold water was added and then storaged in $4^{\circ} \mathrm{C}$ for $15 \mathrm{~min}$. Finally, dilution of the solution (in which red cells were lysed) was made by adding a phosphate buffer solution $(\mathrm{pH}=7.0)$.

A spectophotometer was used for the measurement of SOD activity in $37^{\circ} \mathrm{C}$ and $505 \mathrm{~nm}$. The percentage of inhibition was calculated on the basis of the absorptions measured. The concentrations of SOD activity (in units $/ \mathrm{ml}$ ) were calculated by using a model curve of solution with known concentrations. The normal values proposed by the manufacturer are: $164-240 \mathrm{u} / \mathrm{ml}$.

\section{Statistical analysis}

The Statistical Program for Social Science (SPSS version 17) was used. Paired $t$-test was performed between SOD on admission and SOD on discharge. Pearson bivariate correlation was used for comparison between SOD on admission and $\mathrm{FEV}_{1}$ on admission and also between SOD on admission and $\mathrm{PaO}_{2}$ on discharge. A $p$ value lower than 0.05 was considered statistically significant.

\section{Results}

Mean $\mathrm{FEV}_{1}$ (\% predicted) on admission was $44.02 \pm 12.43$ and on discharge $65.55 \pm 10.97$. Mean SOD activity on admission was $43.64 \pm 31.78$ units $/ \mathrm{ml}$ and on discharge $96.16 \pm 54.05$ units $/ \mathrm{ml}$ (table 1).

\begin{tabular}{lc} 
Table 1. - Demographics, pulmonary function and \\
SOD data \\
\hline No of patients & 38 \\
$\quad$ Males & 33 \\
Females & 5 \\
Age & $56 \pm 2.8$ \\
Atopy & 17 \\
Inhaled steroids & 18 \\
FEV $_{1}$ on admission $(\%$ pred) & $44.02 \pm 12.43^{*}$ \\
FEV $_{1}$ on discharge $(\%$ pred) & $65.55 \pm 10.97$ \\
$\mathrm{PO}_{2}$ on admission (mmHg) & $61.99 \pm 8.82 *$ \\
$\mathrm{PO}_{2}$ on discharge (mmHg) & $78.18 \pm 8.89$ \\
$\mathrm{SOD}_{\text {on }}$ admission (units/ml) & $43.64 \pm 31.78 *$ \\
SOD on discharge (units $/ \mathrm{ml})$ & $96.16 \pm 54.05$ \\
\hline * Statistically significant differences compared with discharge.
\end{tabular}


A statistically significant increase of SOD on discharge was observed compared to that on admission ( $p<0.001$ paired t-test) (fig. 1). A statistically significant positive correlation was observed between SOD and $\mathrm{FEV}_{1}$ (\% of predicted) on admission $(r=0.57, p<0.001)$ but not on discharge (Pearson correlation two tailed significant) (fig. 2). A statistically significant positive correlation was observed between SOD on discharge and $\mathrm{PaO}_{2}$ on discharge $(\mathrm{r}=0.53, \mathrm{p}=0.001)$ but not on admission (fig. 3). Finally a statistically significant correlation was observed between SOD on admission and $\mathrm{PaO}_{2}$ on discharge $(r=0.55, p<0.001)$ (fig. 4).

No other statistically significant correlation was observed between SOD and any other respiratory function parameters or other severity criteria.

\section{Discussion}

This study demonstrated decreased erythrocyte superoxide dismutase activity in patients with severe exacerbation of bronchial asthma. This activity was significantly improved once the respiratory parameters of asthmatic patients returned to near normal values. This finding was also supported by the positive correlation found between SOD activity and $\mathrm{FEV}_{1}$ on the day of admission and between SOD activity on admission and $\mathrm{PaO}_{2}$ on discharge, as well as SOD on discharge and $\mathrm{PaO}_{2}$ on discharge.

Many observations suggest that levels of oxidative stress are increased not only in the lungs but also in the circulation of adults and children with asthma [1]. Conversely, several reports have found alterations in different antioxidants in asthma and recent literature in this field seems to indicate conflicting findings. For instance, lower erythrocyte or serum SOD activity has been reported in asymptomatic mild adult asthmatics compared with controls $[14,15]$ and lower levels of SOD have been found in children with mild asthma compared with healthy controls [16]. However, increased SOD activity in red blood cells, [10] and higher levels of SOD in mild stable asthmatics have been observed [17]. A plausible explanation for the above discrepancies could be that oxidantantioxidant balance is not significantly affected in mild asthmatics and/or erythrocyte SOD activity is not sensitive to the oxidant-antioxidant balance in this kind of patients [17]. In addition, different results should be expected if levels of SOD (either in red blood cells or serum) were studied instead of the activity of the enzyme.

The effects of asthma exacerbation on the oxidant/antioxidant imbalance have received far less attention. There is evidence of enhanced oxidative stress during exacerbations in the peripheral blood $[18,19]$, but conflicting findings have been reported regarding the antioxidant status. For example, it was observed in a study that loss of SOD activity occurs within minutes of an acute antigen-induced asthmatic response [20], but in another study no significant difference in erythrocyte antioxidant enzyme activities, including SOD, was observed between patients with acute exacerbation and with stable asthma [18]. In the present study, decreased erythrocyte SOD activity was found on admission compared to discharge, and as it was measured twice during the few days of hospitalisation, along with certain clinical and laboratory improvement of all patients at the same period, it seems that asthma attack is accompanied by decreased activity of SOD.

Previous reports show that localised decreases in SOD activity occur within airway epithelial cells, bronchoalveolar lavage fluid, and bron-

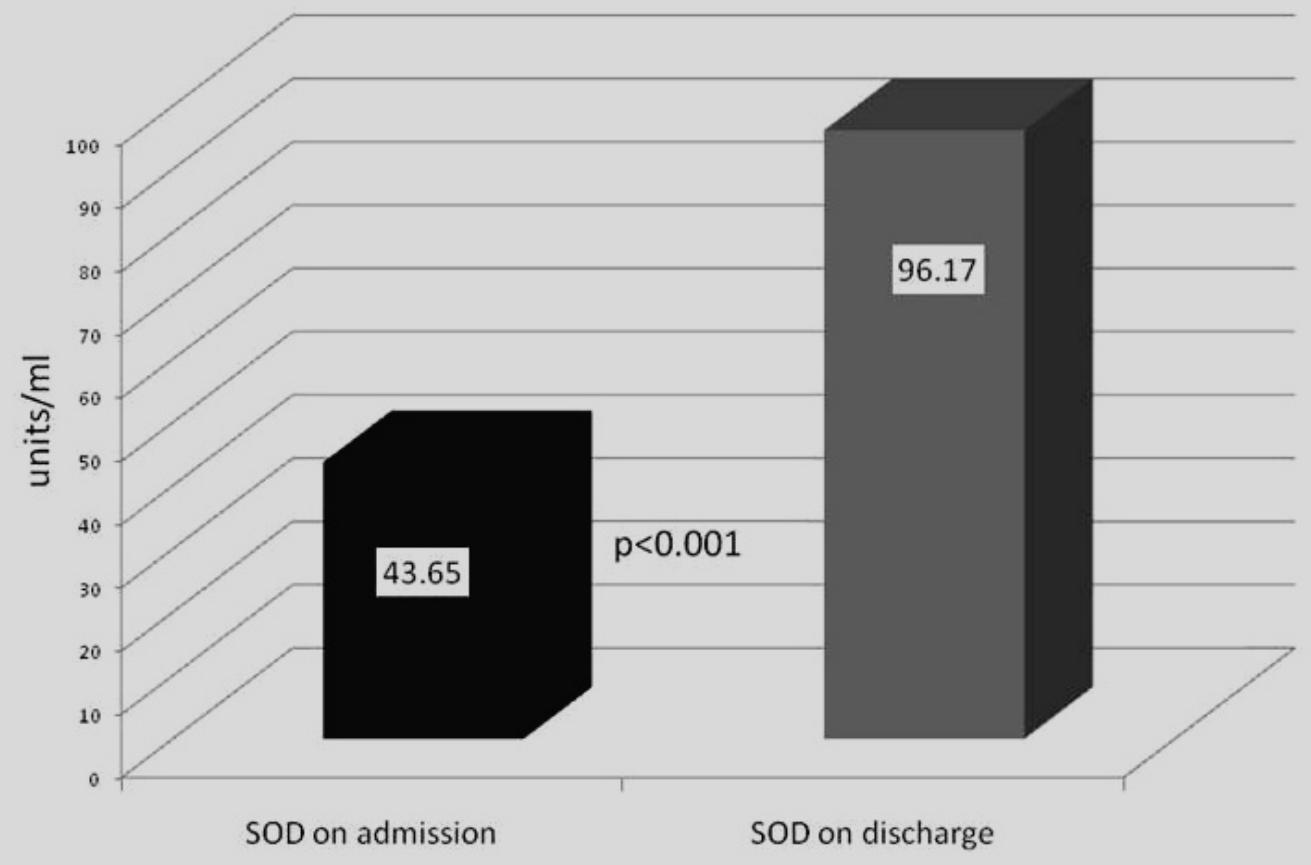

Fig. 1. - SOD on admission and on discharge. 


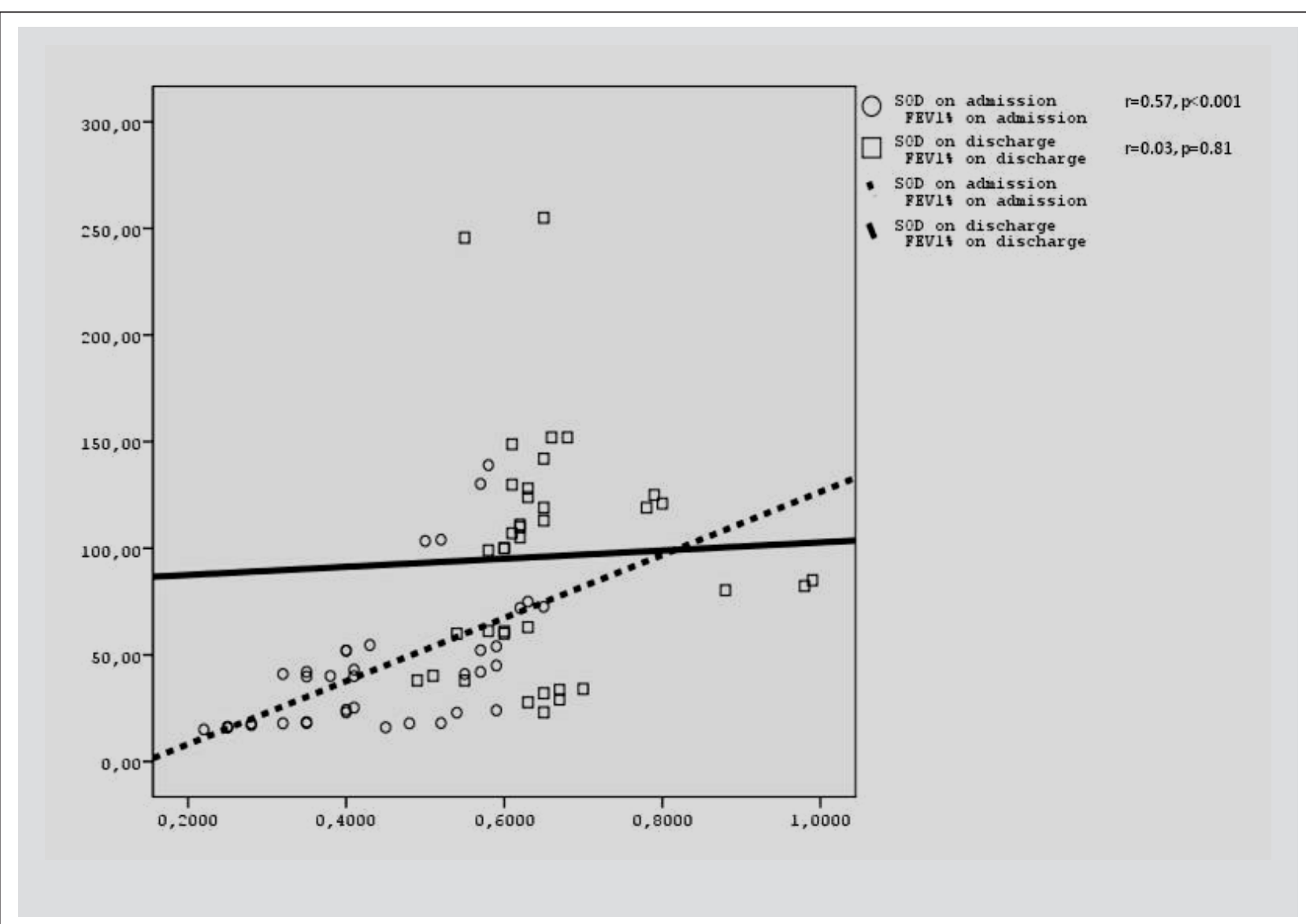

Fig. 2. - Correlation between SOD and $\mathrm{FEV}_{1}$ on admission and on discharge.

choalveolar lavage cells in proportion to airflow limitation and asthmatic exacerbations [20-22]. It has not been clarified yet whether systemic reductions in activity of the antioxidant protective enzyme occur during asthma attack. However, there is evidence that all SOD enzymes are sensitive to oxidative modification and inactivation [23, 24]. In addition, several studies have indicated that increased oxidative stress also occurs in the circulation of asthmatic subjects as there are increased superoxide anion generation by leukocytes $[1,10$, $18,25]$, increased lipid and/or protein peroxidation products $[1,10,17,18]$ and increased reactive oxygen metabolite levels [19]. Loss of blood SOD activity in asthma may thus reflect a greater magnitude and/or ongoing systemic oxidative stress with a consequent greater oxidative modification of SOD systematically [15]. It has also been proposed that oxidative stress may cause inactivation of antioxidant enzymes, such as SOD with consequent increase in apoptosis, shedding of airway epithelial cells and airway remodeling [26].

It has been suggested that corticosteroids have a beneficial effect on antioxidants [27]. Previous studies have shown that treatment with corticosteroids reduces oxidative stress and restores intracellular SOD activity levels in mild asthma [28, 29]. In our study, no correlation between inhaled steroids and SOD activity was found and complements the findings of a previous study [15], thus making it less likely that inhaled steroids influence SOD activity. On the other hand, parenteral corticosteroids were administered in all patients during hospitalisation and all of them improved SOD activity on discharge. Further studies are necessary to evaluate whether high-dose systemic corticosteroids improve antioxidant capacity in asthma.

Wood et al [30] found that SOD activity was negatively associated with asthma severity in patients with mild disease. Kanazawa et al [31] demonstrated an enhanced production of superoxide anion from neutrophils in asthmatic patients. The superoxide anion release was greater in patients with exacerbation of their disease and was also inversely correlated with $\mathrm{FEV}_{1}$. Finally, direct correlation of SOD activity with $\% \mathrm{FEV}_{1}$ has been reported [15]. We also observed similar results between SOD activity and respiratory function parameters such as FEV 1 on admission and $\mathrm{PaO}_{2}$ on discharge, suggesting that decreased SOD activity during asthma exacerbation may not be just an epiphenomenon, but part of the characteristic pathophysiological changes of asthma.

Although markers of oxidative stress in plasma do not directly reflect the local antioxidant capacity in the lungs, other procedures such as bronchoscopy (to obtain bronchoalveolar lavage or bronchial epithelial cells) or condensate of expired air may be practically difficult or impossible to perform in asthmatic patients when they are suffering from an asthmatic attack.

In conclusion, the results of our study indicate the presence of decreased plasma antioxidant capacity during an asthma attack, through decreased erythrocyte SOD activity, and improvement in SOD activity after the acute phase, even though it 


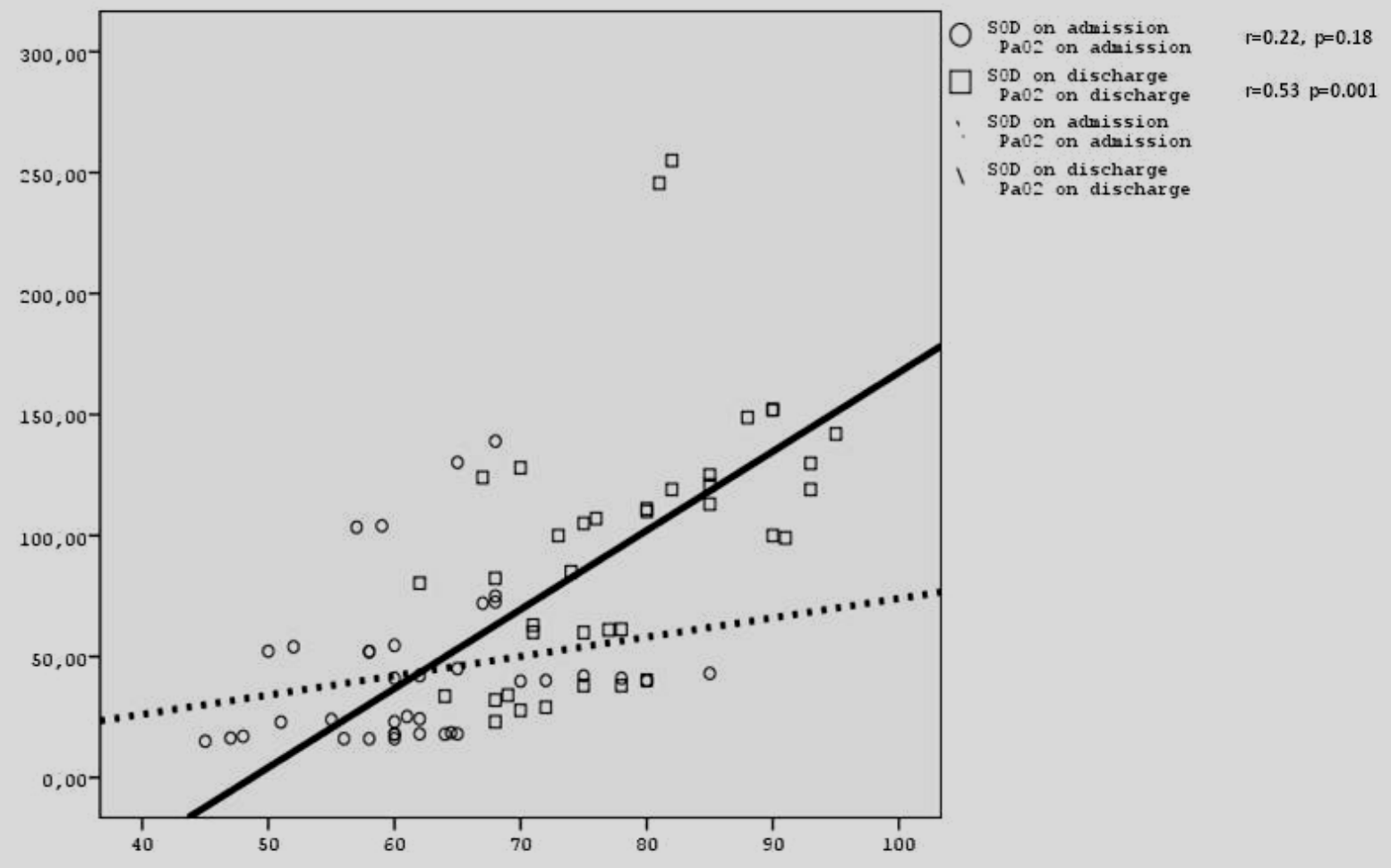

Fig. 3. - Correlation between $\mathrm{SOD}$ and $\mathrm{PaO}_{2}$ on admission and on discharge.

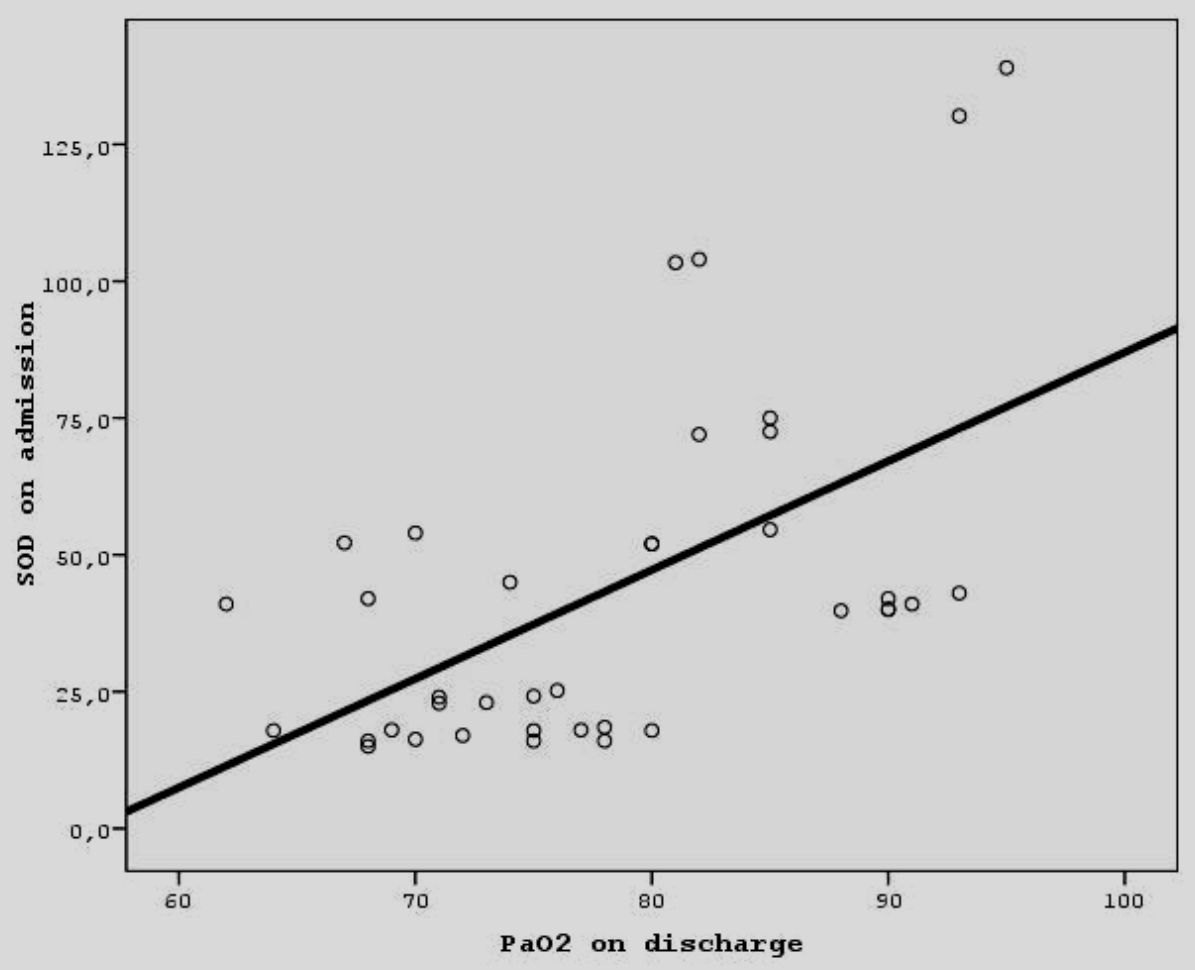

$r=0.55, p<0.001$ 
did not meet the normal values. Although respiratory tract infection was the most frequent cause of the exacerbation (which is an additional oxidative load), positive correlations with severity criteria such as $\mathrm{FEV}_{1}$ on admission and $\mathrm{PaO}_{2}$ on discharge were also found. These observations, along with others previously reported, endorse the correlation between disease severity and oxygen radical production in asthma patients. Therefore, it seems that measurement of erythrocyte SOD activity could be a quite simple and useful tool in the evaluation of the severity of an asthma attack as well as a blood biomarker to monitor remission after acute attack. However, further studies and data would be useful to explore whether this factor or other antioxidants could be used as additional severity criteria or even prognostic factors and also to clarify the benefit of supplementary administration of antioxidants.

\section{References}

1. Mak JCW, Chan-Yeung MMW. Reactive oxygen species in asthma. Curr Opin Pulm Med 2006; 12: 7-11.

2. Bowler RP. Oxidative stress in the pathogenesis of asthma. Curr Allergy Asthma Rep 2004; 4: 116-122.

3. Dworski R. Oxidant stress in asthma. Thorax 2000; 55 (Suppl 2): S51-S53.

4. Hoshino T, Okamoto M, Takei S, Sakazaki Y, Iwanaga $\mathrm{T}$, et al. Redox-regulated mechanisms in asthma. Antioxid Redox Signal 2008; 10: 769-83.

5. Reddy SF. The antioxidant response element and oxidative stress modifiers in airway diseases. Curr $\mathrm{Mol}$ Med 2008; 8: 376-83.

6. Rahman I, Biswas SK, Kode A. Oxidant and antioxidant balance in the airways and airways diseases. Eur $J$ Pharmacol 2006; 533: 222-39.

7. Nagata M. Inflammatory cells and oxygen radicals. Curr Drug Targets Inflamm Allergy 2005; 4: 503-4.

8. Kinnula VL, Crapo JD. Superoxide dismutases in the lung and human lung diseases. Am J Respir Crit Care Med 2003; 167: 1600-1619.

9. Evans P, Haliwell B. Micronutrients: oxidant/antioxidant status. Brit J Nutr 2001; 85: S67-S74.

10. Nadeem A, Chhabra SK, Masood A, Raj HG. Increased oxidative stress and altered levels of antioxidants in asthma. J Allergy Clin Immunol 2003; 111: 72-78.

11. Mak JC, Leung HC, Ho SP, Law BK, Lam WK, et al. Systemic oxidative stress and antioxidative status in Chinese patients with asthma. J Allergy Clin Immunol 2004; 114: 260-264.

12. Katsoulis K, Kontakiotis T, Leonardopoulos I, et al. Serum total antioxidant status in severe exacerbation of asthma: correlation with the severity of the disease. $J$ Asthma 2003; 40: 847-854.

13. Global Strategy for Asthma Management and Prevention, Global Initiative for Asthma (GINA) 2008. http://www.ginasthma.org

14. Tekin D, Sin BA, Mungan D, et al. The antioxidative defense in asthma. J Asthma 2000; 37: 59-63.
15. Comhair SA, Ricci KS, Arroliga M, et al. Correlation of systemic superoxide dismutase deficiency to airflow obstruction in asthma. Am J Respir Crit Care Med 2005; 72: 306-13.

16. Sackesen C, Ercan H, Dizdar E, et al. A comprehensive evaluation of the enzymatic and nonenzymatic antioxidant systems in childhood asthma. J Allergy Clin Immunol 2008; 122: 78-85.

17. Hanta I, Kuleci S, Canacankatan N, Kocabas A. The oxidant-antioxidant balance in mild asthmatic patients. Lung 2003; 181: 347-52.

18. Nadeem A, Raj HG, Chhabra SK. Increased oxidative stress in acute exacerbations of asthma. J Asthma 2005; 42: $45-50$

19. Suzuki S, Matsukura S, Takeuchi H, et al. Increase in reactive oxygen metabolite level in acute exacerbation of asthma. Int Arch Allergy Immunol 2008; 146: 67-72.

20. Comhair SA, Bhathena PR, Dweik RA, Kavuru M, Erzurum SC. Rapid loss of superoxide dismutase activity during antigen-induced asthmatic response. Lancet 2000; 355: 624.

21. Smith LJ, Shamsuddin M, Sporn PH, Denenberg M, Anderson J. Reduced superoxide dismutase in lung cells of patients with asthma. Free Rad Biol Med 1997; 22: 1301-1307.

22. Smith LJ, Shamsuddin M, Sporn PH, Denenberg M, Anderson J. Superoxide dismutase inactivation in pathophysiology of asthmatic airway remodelling and reactivity. Am J Pathol 2005; 166: 663-674.

23. Sharonov BP, Churilova IV. Inactivation and oxidative modification of $\mathrm{Cu}, \mathrm{Zn}$ superoxide dismutase by stimulated neutrophils: the appearance of new catalytically active structures. Biochem Biophys Res Commun 1992; 189: 1129-1135.

24. Alvarez B, Demicheli V, Duran R, et al. Inactivation of human $\mathrm{Cu}, \mathrm{Zn}$ superoxide dismutase by peroxynitrite and formation of histidinyl radical. Free Rad Biol Med 2004; 37: 813-822.

25. Nagata M. Inflammatory cells and oxygen radicals. Curr Drug Targets Inflamm Allergy 2005; 4: 503-4.

26. Misso NL, Thompson PJ. Oxidative stress and antioxidant deficiencies in asthma: potential modification by diet. Redox Rep 2005; 10: 247-55.

27. Sadowska AM, Klebe B, Germonpré P, De Backer WA. Glucocorticosteroids as antioxidants in treatment of asthma and COPD: new application for an old medication? Steroids 2007; 72: 1-6.

28. Sadowska AM, Klebe B, Germonpré P, De Backer WA. Decreased $\mathrm{Cu}, \mathrm{Zn}-\mathrm{SOD}$ activity in asthmatic airway eprithelium: correction by inhaled corticosteroid in vivo. Am J Physiol 1997; 272: 148-54.

29. Majori M, Vachier I, Godard P, et al. Superoxide anion production by monocytes of corticosteroid-treated asthmatic patients. Eur Respir J 1998; 11: 13-8.

30. Wood LG, Fitzgerald DA, Gibson PG, Cooper DM, Garg ML. Lipid peroxidation as determined by plasma isoprostanes is related to disease severity in mild asthma. Lipids 2000; 35: 967-74.

31. Kanazawa H, Kurihara N, Hirata K, Takeda T.. The role of free radicals in airway obstruction in asthmatic patients. Chest 1991; 100: 1319-22. 